184

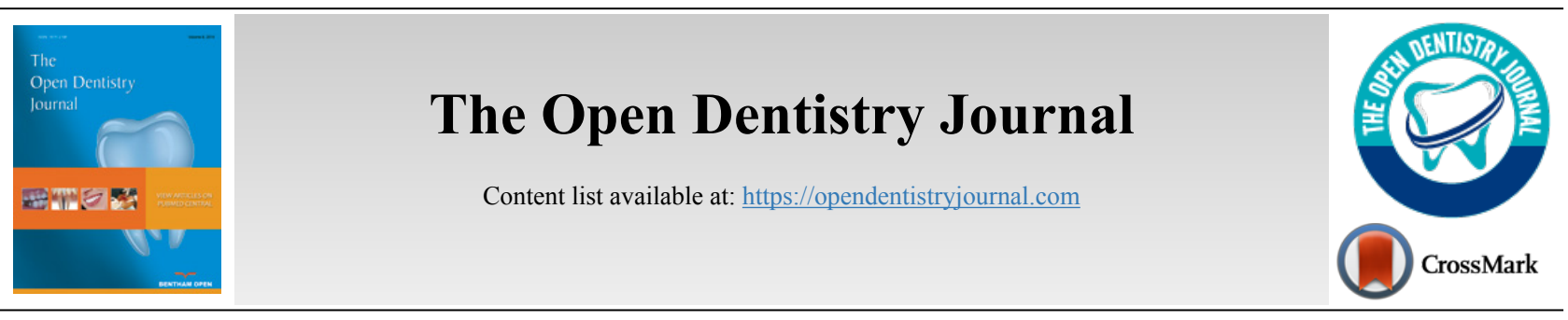

RESEARCH ARTICLE

\title{
The Prevalence and Etiology of Molar-Incisor Hypomineralization among Female School Children
}

\author{
Ghofran S. Sulaimani ${ }^{1}$, Lama O. Hejazi ${ }^{1}$ and Hanadi S. Lingawi ${ }^{1, *}$ \\ ${ }^{I}$ Department of Preventive Dentistry, College of Dentistry, Umm Al-Qura University, Makkah, Saudi Arabia
}

\begin{abstract}
:
Background:

Molar-Incisor Hypomineralization (MIH) is a developmental defect affecting teeth. The prevalence of MIH and its clinical implications form a challenge for patients and dentists.

Objective:

The purpose of this study was to determine the prevalence of MIH among female school children in Makkah, Saudi Arabia.

Methods:

A cross-sectional study was conducted including 7- to 9-year-old school girls from the public and private sectors. Invitations to participate in the study were sent to the parents of 1,000 randomly selected children, along with questionnaire and consent form. All included children were examined by two dentists who had been previously calibrated in the assessment of MIH $(\mathrm{K}=0.9)$, which was diagnosed according to the European Academy of Paediatric Dentistry 2003 criteria. Data analysis was done using SPSS V.22.0 and various statistical tests were conducted to determine significant differences at $\mathrm{P}<0.05$.

Results:

A total of 711 children participated in this study, with a response rate of $82.2 \%$. The prevalence rates of MIH and Molar Hypomineralization (MH) were $20.96 \%$ and $26.58 \%$, respectively. The most common form of MIH was the mild form ( $87.24 \%)$, while 18 children (12.08\%) were diagnosed with the moderate form. A single child $(0.67 \%)$ was diagnosed with the severe form. No statistically significant associations were found between $\mathrm{MIH} / \mathrm{MH}$ and prenatal, perinatal or postnatal factors.

Conclusion:

The prevalence of MIH in school girls in Makkah was $20.96 \%$. Despite its high prevalence, the severity of MIH was relatively mild. The etiology of MIH remains unclear and warrants further investigation in future studies.
\end{abstract}

Keywords: Molar-incisor hypomineralization, School children, Enamel hypoplasia, Dental anomalies, Prevalence, Prevalence rate.

\begin{tabular}{l|l|l|l} 
Article History & Received: October 21, 2020 & Revised: February 3, 2021 & Accepted: February 22, 2021
\end{tabular}

\section{INTRODUCTION}

Since the 1970s, many cases of developmental defects localized to the First Permanent Molar(s) (FPMs) and incisor(s) have been reported by dentists [1]. Clinical descriptions of these reported cases included opaque enamel with colour ranging from white to yellowish brown and areas with no mineralization or hypomineralization of enamel that varied according to condition severity. Enamel breakdown indicates more severe conditions.

\footnotetext{
* Address correspondence to this author at the Department of Preventive Dentistry, College of Dentistry, Umm Al-Qura University, P.O. Box 14405, Makkah 22915, Saudi Arabia; Tel. +966 505682076;

E-mail: hslingawi@uqu.edu.sa
}

In 2001, the term Molar-Incisor Hypomineralization (MIH) was introduced by Weerheijm et al. [2]. Molar-Incisor Hypomineralization is defined as a hypomineralization of systemic origin of one to four FPMs that is frequently associated with affected incisors [2]. The lesions in the FPMs are often associated with maxillary incisors and more rarely with mandibular incisors. When only FPMs are affected while the incisors are spared, the condition is called Molar Hypomineralization (MH) [2].

MIH is usually attributed to systemic insults during the mineralization of the FPM and incisor crowns in the first few years of a child's life [2]. The severity of MIH (mild, moderate or severe) may vary between different teeth in the same 
individual [3] and is a challenging condition for dentists as well as patients. It is usually associated with serious complications such as severe discomfort, hypersensitivity, shooting pain, early loss of fillings and post-eruptive breakdown due to the hypomineralized tooth structure. Children affected with MIH are more susceptible to dental caries and have more Decayed, Missing and Filled Teeth (DMFT) compared to other children with different types of enamel defects [4].

MIH has received increasing attention in the literature in recent years, particularly in Europe, Australia and South America. The worldwide prevalence of MIH has been reported to range from $2.3 \%$ in Egypt [5] to $40.2 \%$ in Brazil [6]. The wide variation in MIH prevalence rates may be attributed not only to the different geographical regions studied but also to the assessment of different age groups and the use of different indices and criteria for the evaluation of hypomineralization; this has precluded valid comparisons across the studies.

In terms of the Middle East region, prior studies have reported the prevalence of MIH in Libya [7], Egypt [5], Jordon [8], Lebanon [9] and Iraq [10, 11]. Very few studies have been conducted on the Arabian Peninsula. Examples include studies conducted in Bahrain [12] and the United Arab Emirates [13]. In addition, two studies have been conducted among school children in Saudi Arabia; these studies reported MIH prevalence rates of $8.6 \%$ and $40.7 \%$ in the cities of Jeddah [14] and Riyadh [15], respectively. Notably, these two studies utilised different protocols for MIH assessment and included participants with different age ranges. Furthermore, the study conducted in Jeddah utilised a small sample size; thus, caution must be used in generalising its results to the nation as a whole.

While the number of studies aimed at elucidating the etiological factors of MIH has increased in recent years, the results have been inconclusive [16 - 18]. Nevertheless, it is widely accepted that the etiology of MIH is multifactorial and includes both genetic and environmental factors [17, 18]. Environmental postnatal factors include fevers, chickenpox, tonsillitis, asthma, repeated ear infections, allergies and the repeated use of antibiotics during the first 4 years of life. Prenatal factors include illnesses during pregnancy, birth complications and premature birth.

The dental treatment of MIH/MH cases has proven to be a challenge for both patients and dentists. The porosity of dental enamel leads to pulpal inflammation, and the increased number of nerve endings in dentinal tubules results in sensitivity and pain. Additionally, there are often difficulties in achieving adequate anesthesia. As a result, children with $\mathrm{MIH} / \mathrm{MH}$ usually experience dental fear and anxiety. Therefore, it is important to determine the prevalence of $\mathrm{MIH}$ in a given population, as this will facilitate the development and implementation of policies for its prevention and management. The aim of this study was to determine the prevalence of MIH among female school children in Makkah, Saudi Arabia, and to investigate the possible associated etiological factors.

\section{MATERIALS AND METHODS}

\subsection{Study Design and Ethical Considerations}

The study protocol was approved by the Institutional
Review Board (IRB) of the College of Dentistry, Umm AlQura University (IRB no. 87-18). This study utilised a crosssectional design and was conducted among 10 primary private and public schools for girls in Makkah, Saudi Arabia. Schools from both private and public sectors were selected to ensure that all socio-economic statuses are included in the study. The schools were selected through stratified random selection to cover different districts. The 10 schools were invited to participate in the study via official letters from the Regional Director of the School Health Department in Makkah.

After a thorough review of the literature, a questionnaire was carefully constructed and designed by the authors to document possible etiological factors associated with $\mathrm{MIH} /$ MH. It covered four domains: (1) demographic data, (2) maternal health and medications administered during pregnancy, (3) delivery history and (4) the child's medical history during her first 3 years of life (Fig. 1).

Prior to questionnaire distribution, a pilot study to test the precision of the survey questions was conducted with 10 participants. Based on their responses, 4 survey questions were modified, and the format was edited for clarity and comprehensibility. A second pilot study was conducted with another 10 participants to confirm the feasibility, validity and reliability of the questionnaire. A total of 1,000 parental consent forms and questionnaires were distributed to the participating schools. The number of schools and sample size was adjusted to the time limitations of the study, the limited number of clinical examiners ( 2 dentists) and the research budget.

The sample inclusion criteria comprised the following: (1) females aged 7-9 years, (2) born and brought up in Saudi Arabia and (3) children having at least one erupted or partially erupted FPM. Participants were excluded if they had (1) generalized opacities present on all teeth rather than those limited to the FPMs and permanent incisors such as in case of amelogenesis imperfecta, (2) cases of fluorosis, which is usually diffuse and generalized affecting other than the target teeth, (3) defects in the permanent dentition due to prior dental trauma/ infection in the primary dentition.

\subsection{Clinical Examination and Diagnosis}

The clinical examinations were performed by two general dentists who had been previously trained and calibrated (using photographs of clinical cases) in the identification of MIH according to the European Academy of Paediatric Dentistry (EAPD) 2003 criteria [19]. MIH severity was determined according to the Wetzel and Reckel criteria (Table 1) [3], and inter-examiner reliability was determined using Cohen's kappa coefficient prior to study commencement; a high level of reliability $(\mathrm{K}=0.9)$ was documented. Children were examined at their respective school premises by one of the two trained examiners. The intraoral examinations were carried out within a classroom setting and conducted with a headlight and disposable dental examination kit that contained the following items: a dental mirror, tweezers, dental explorer and multiple cotton rolls. 


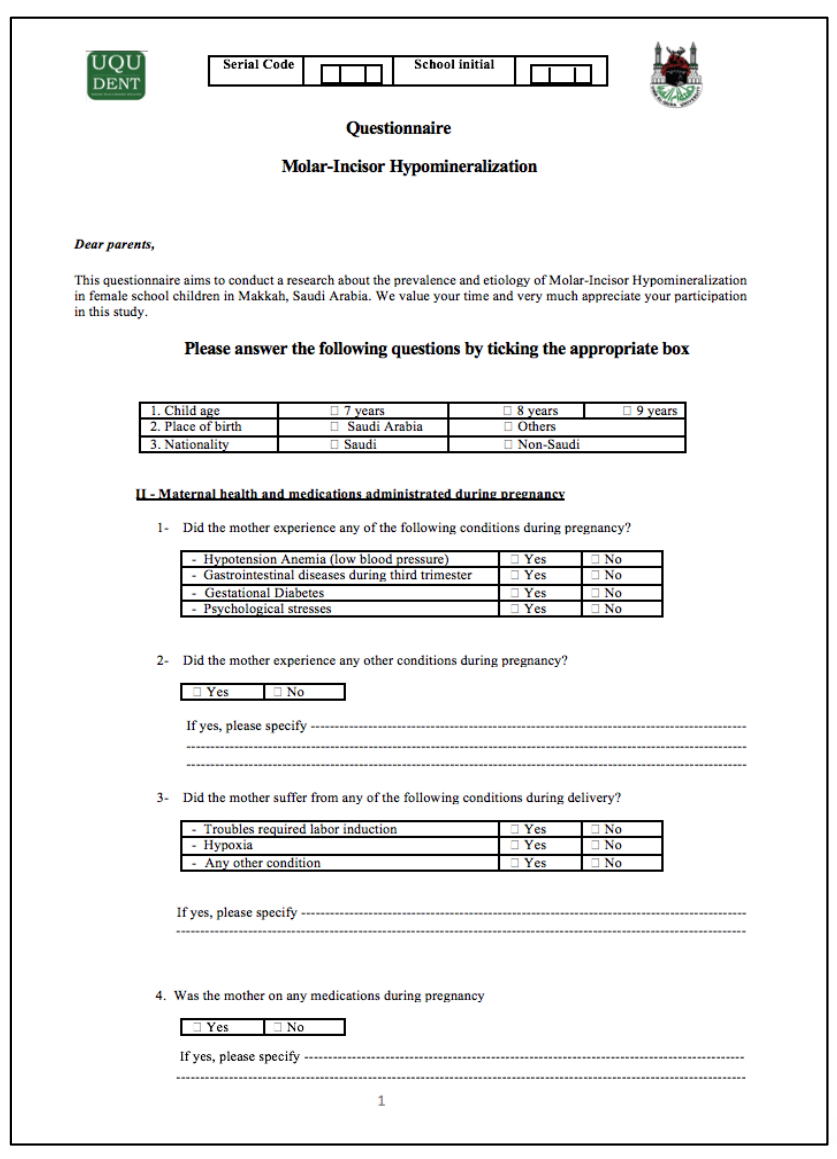

Fig. (1). Sample of the questionnaire used in the study.

Table 1. Wetzel and Reckel scale of MIH severity.

\begin{tabular}{|c|c|c|}
\hline Degree 1 (mild) & Degree 2 (moderate) & Degree 3 (severe) \\
\hline $\begin{array}{l}\text { Isolated white and } \\
\text { cream to yellowish- } \\
\text { brown discolorations } \\
\text { on the occlusal } \\
\text { surface and coronal } \\
\text { third of the crown. }\end{array}$ & $\begin{array}{l}\text { Hypomineralized } \\
\text { yellowish-brown } \\
\text { enamel on the } \\
\text { majority of the cusps, } \\
\text { but with only a slight } \\
\text { loss of substance. }\end{array}$ & $\begin{array}{c}\text { Large-scale mineral } \\
\text { deficiency with distinct } \\
\text { yellowish-brown } \\
\text { discolorations and } \\
\text { defects in crown } \\
\text { morphology resulting } \\
\text { from extensive loss of } \\
\text { enamel. }\end{array}$ \\
\hline
\end{tabular}

\subsection{Statistical Analysis}

Data were entered into a Microsoft Excel spreadsheet and analysed using the Statistical Package of Social Sciences program (SPSS 22.0, Inc., Chicago, USA). Descriptive statistics were used to describe the data in which frequency and percentage for categorical variables were determined. Patient characteristics were presented descriptively, and the frequencies of potential etiological factors were compared between children with MH and MIH using Pearson's chisquare test. A critical probability value (P-value) of $<0.05$ was used as the cut-off level for statistical significance.

\section{RESULTS}

Parents of 865 children provided written informed consent and completed/returned the questionnaires (response rate $=$ $86.5 \%$ ); however, not all of the children were present on the

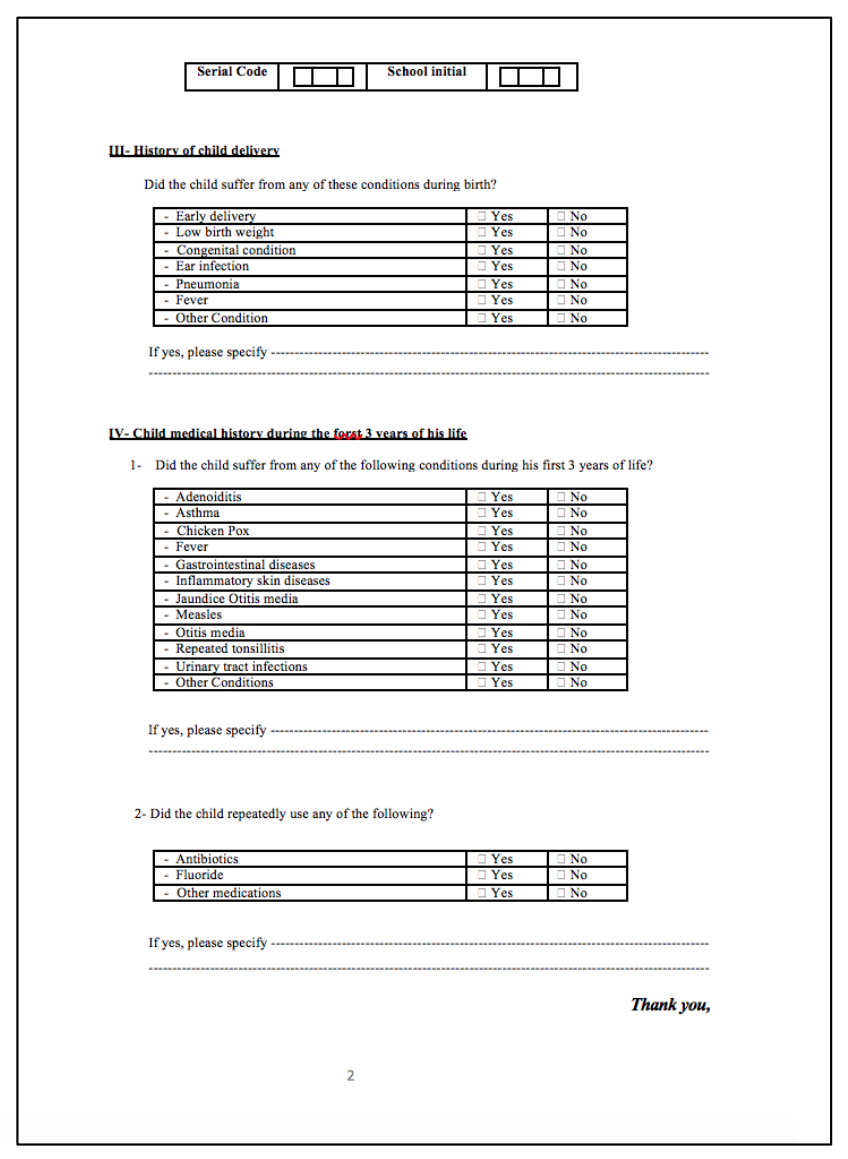

day of the clinical examinations, resulting in a final sample size of 711 . The mean age of the recruited children was $8.1 \pm 0.73$; $536(75.39 \%)$ were Saudis and 175 (24.61\%) were non-Saudis.

A total of 149 children $(20.96 \%)$ were diagnosed with MIH, while 189 (26.58\%) were diagnosed with MH. Among Saudi children, the prevalence rates of MIH and MH were $21.83 \%(\mathrm{n}=117)$ and $27.24 \%(\mathrm{n}=146)$, respectively (Fig. 2$)$. Lower prevalence rates of MIH $(18.29 \% ; \mathrm{n}=32)$ and $\mathrm{MH}$ $(24.57 \% ; \mathrm{n}=43)$ were observed among non-Saudi children (Fig. 3).

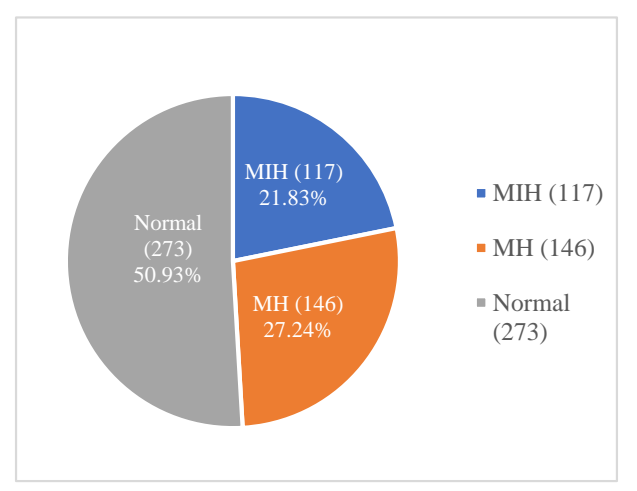

Fig (2). The prevalence of MIH and MH reported among Saudi female school children (MIH: molar incisor hypomineralization; MH: molar hypomineralization). 
In terms of MIH severity, 130 children $(87.25 \%)$ were diagnosed with mild $\mathrm{MIH}$, while 18 children (12.08\%) were diagnosed with moderate MIH. A single child $(0.67 \%)$ was diagnosed with severe MIH (Table 2). No severe cases of MH were documented; 187 (98.94\%) and 2 children (1.06\%) were diagnosed with mild and moderate $\mathrm{MH}$, respectively.

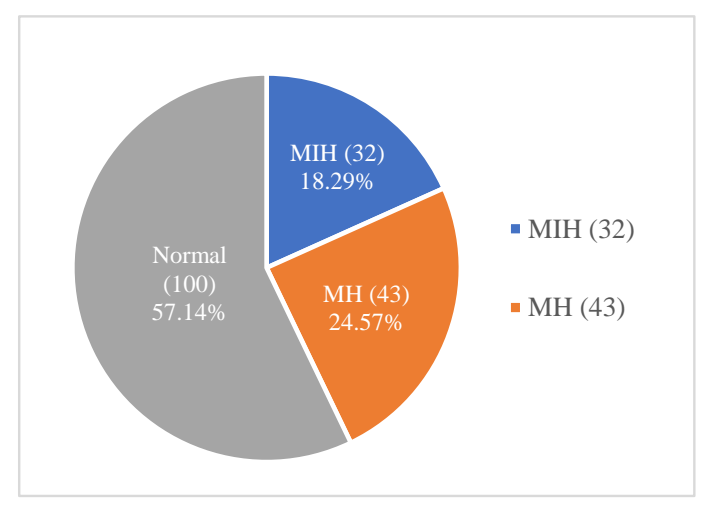

Fig. (3). The prevalence of MIH and MH reported among non-Saudi female school children (MIH: molar incisor hypomineralization; $\mathrm{MH}$ : molar hypomineralization).

No significant associations were found between $\mathrm{MIH} / \mathrm{MH}$ and pre-natal factors (i.e., birth complications, illnesses during pregnancy or birth complications) or illnesses affecting the child during her first 3 years of life (e.g. fever, jaundice, otitis media, or frequent tonsillitis) (Table 3). Frequent antibiotic use was more commonly reported for children with $\mathrm{MH}(46.5 \%)$ compared to children with MIH (42.9\%); nevertheless, this did not reach statistical significance $(p$ value $=0.074)($ Table 3$)$.

\section{DISCUSSION}

Prevalence studies play a major role when strategies and policies need to be developed to prevent/treat certain conditions. This study represented the first MIH prevalence and etiology study conducted in the city of Makkah and the third of its type in Saudi Arabia [14, 15].

Both the response $(86.5 \%)$ and final participation rates $(82.2 \%)$ were good; therefore, the recruited sample was considered to be representative of the target population. The age range chosen in this study (7-9 years old) was considered suitable for MIH assessment, as the four FPMs would have erupted by this age in most children. A similar age range has been used in other studies, such as in Zawaideh et al.'s study of MIH in Jordanian children, in Petrou et al.'s study of MIH prevalence among German school children, and in Mittal et al. $[8,20,21]$. This age range criterion also minimized the risk that the areas of enamel hypomineralization would be masked by subsequent dental caries or restorations. The selection criteria of this study excluded children with generalized opacities present on all teeth, fluorosis or defects in the permanent dentition due to prior dental trauma/ infection in the primary dentition, as these conditions represent a grey area in the diagnoses, where $\mathrm{MIH} / \mathrm{MH}$ might be masked or misdiagnosed with other conditions. For instance, diffuse opacity may be caused by the intake of a high concentration of fluoride and should not, according to Weerheijm et al. [19], be classified as MIH.

Table 2. Prevalence of MIH severity degrees in the studied sample.

\begin{tabular}{|c|c|c|c|c|}
\hline MIH severity & Frequency $(\boldsymbol{n})$ & Percentage (\%) & $\boldsymbol{X}^{2}$ & $\boldsymbol{p}$ value \\
\cline { 1 - 3 } Mild & 130 & 87.25 & 748.203 & 0.000 \\
\cline { 1 - 3 } Moderate & 18 & 12.08 & & \\
\cline { 1 - 3 } Severe & 1 & 0.67 & & \\
\cline { 1 - 3 } TOTAL & 149 & 100.00 & & \\
\hline
\end{tabular}

In an effort to ensure the consistency and reliability of the clinical assessments, all examinations were carried out by two general dentists who had been previously trained and calibrated.

The results of our study showed that 338 children (47.5\%) of all examined children had hypomineralization of at least one examined tooth. The prevalence of MIH in our study was $20.96 \%$, which was greater than that reported by Allazzam et al. $(8.6 \%)$ in a study conducted in Jeddah, which is located on Saudi Arabia's west coast [14]. This difference may have been attributed to their use of a convenience sample obtained at a single university teaching hospital, as well as a small sample size of 267 children. Indeed, Elfrink et al. [22] suggested that a minimum random sample size of 300 participants would be required for an accurate estimation of $\mathrm{MIH}$ prevalence. AlHammad et al. [15] reported a much higher prevalence of MIH $(40.7 \%)$ in the city of Riyadh, located in the middle of Saudi Arabia. While this may be due to true regional variation, the effect of differences in study methodology must also be acknowledged; this emphasises the importance of conducting a national survey for the accurate estimation of MIH prevalence in Saudi Arabia.

Based on the results of the present study, MIH in Saudi Arabia was considered to be moderately prevalent relative to other countries around the world, such as Brazil (40.2\%) [6], Denmark (37.5\%) [23], Finland (25\%) [24] and Egypt (3.2\%) [5]. However, studies conducted in the Middle East region have consistently reported lower prevalence rates; examples include Libya (2.9\%) [7], Jordon (17.6\%) [8], Bahrain (17.6\%) [12] and Iraq $(18.6 \%)[11,12]$. The only country in the Gulf region with a higher reported MIH prevalence than the present study, was the United Arab Emirates (34.4\%) [13]; this may be attributed to the differences in inclusion criteria between both studies and the use of a smaller sample size (less than half that of the present study).

This study is considered one of the very few studies that studied the prevalence of both MIH and MH in the same population. Most of the studies investigated the prevalence of MIH. The results of our study showed a higher prevalence of MIH (20.96\%) than MH (26.58\%) in the studied sample, which goes in agreement with the results reported by Hussien et al. [13]. Their results reported that the prevalence of $\mathrm{MH}$ was higher than MIH (65.6\% and 34.4\%), respectively. 
Table 3. The association between possible etiological factors and the prevalence of MIH/ MH.

\begin{tabular}{|c|c|c|c|c|c|c|c|}
\hline \multirow[t]{2}{*}{ Condition } & \multicolumn{2}{|c|}{ MIH } & \multicolumn{2}{|c|}{ МH } & \multicolumn{2}{|c|}{ Not Affected } & \multirow[t]{2}{*}{$p$ Value } \\
\hline & Frequency $(n)$ & Percent (\%) & Frequency $(n)$ & Percent (\%) & Frequency $(n)$ & Percent (\%) & \\
\hline Birth complications & 9 & 6.04 & 14 & 7.41 & 25 & 6.70 & 0.574 \\
\hline Repeated attacks of tonsillitis & 41 & 27.52 & 64 & 33.86 & 120 & 32.17 & 0.769 \\
\hline Otitis media & 18 & 12.08 & 24 & 12.70 & 49 & 13.14 & 0.320 \\
\hline Skin allergy & 22 & 14.67 & 38 & 20.11 & 79 & 21.18 & 0.211 \\
\hline Fever & 70 & 46.98 & 101 & 53.44 & 189 & 50.67 & 0.303 \\
\hline Asthma & 20 & 13.42 & 44 & 23.28 & 87 & 23.32 & 0.283 \\
\hline Jaundice & 12 & 8.05 & 18 & 9.52 & 35 & 9.38 & 0.323 \\
\hline Repeated antibiotic intake & 64 & 42.90 & 88 & 46.56 & 189 & 50.70 & 0.074 \\
\hline Total & 149 & 100 & 189 & 100 & 373 & 100 & \\
\hline
\end{tabular}

MH: Molar Hypomineralization; MIH: Molar Incisor Hypomineralization.

The finding that the majority of the MIH and MH cases were of the mild form is in agreement with the results of other studies conducted among the same age group $[8,20]$. However, this might be age-related. It has been well-established that hypomineralized teeth are weaker and more susceptible to posteruptive enamel breakdown and dental caries. Hence, an increase in hypomineralization severity has been found to be associated with an increase in age $[25,25]$.

It is notable that the prevalence rates of caries and fluorosis are high in the western region of Saudi Arabia [26, 27], as both conditions hinder the assessment of MIH. In order to address this issue, we targeted a younger age group (7-9 years old) in the present study; this allowed for the assessment of permanent teeth soon after their eruption, thus minimizing the probability of existing caries and restorations. Similar to previous studies $[8,13]$, we observed a higher prevalence of MH $(26.58 \%)$ than MIH (20.96\%). This result may have been attributed to the earlier completion of molar formation and calcification compared to incisors [28 - 30].

In terms of the investigation of possible etiological factors for hypomineralization, 663 children did not have birth complications nor hypomineralization; 25 had birth complications but no hypomineralization; and 23 had birth complications and hypomineralization. Almost $47.9 \%$ of our sample population who had birth complications also had hypomineralization. It is justifiable that $\mathrm{MH}$ had higher association with birth complications (14 children) than MIH (9 children). This is because the first permanent molars begin mineralizing around birth, while the mineralization of the first permanent incisors occurs during the first year of life. However, the results of the present study indicated no statistically significant association between prenatal/perinatal factors and MIH/MH [31, 32]. Specifically, two prior studies have also reported the lack of a significant association between birth complications and $\mathrm{MIH} / \mathrm{MH}[18,32]$, supporting the results of our study. On the other hand, childhood illnesses during the first 3 years of life have been reported to be more common among children with MIH or MH [14, 32]. For instance, fever and asthma in the first 3 years of life have been reported to be associated with MIH [33 - 35]. Nevertheless, no significant association was found between $\mathrm{MIH} / \mathrm{MH}$ and either fever or asthma in the present study.
To date, the relationship between $\mathrm{MIH} / \mathrm{MH}$ and repeated use of antibiotics in the first 3 years of life has been controversial. There is also uncertainty about whether the potential effects on hypomineralization are limited to amoxicillin antibiotics or whether all antibiotic types may be implicated. In the present study, the association between hypomineralization and frequent antibiotic use during the first 3 years of life did not reach statistical significance. This was supported by a previous study conducted in Spain, which did not find a significant relationship between antibiotic consumption and MIH/MH [26]. On the other hand, a study conducted in the United Kingdom reported a significant association between MIH and the frequent use of amoxicillin between the ages of 3 and 3.9 years [18]. This result has remained controversial, as other evidence has suggested that the type of antibiotic is not as important as the timing of administration (i.e. during the first 3 years of life) [10]. At any rate, it is difficult to ascertain whether hypomineralization is associated with the prescription of antibiotics to treat an existing infection or the effects of the infection itself.

The main strength of the present study was the large sample size employed, which was the largest to date among studies conducted in the Arabian Gulf countries. A possible limitation was that the questionnaires were self-completed by the parents, and no interviewer was utilized to obtain the responses. This may have compromised the reliability of the answers. Furthermore, the recruited sample was limited to females; a future study utilizing an identical protocol in boys' schools is currently being planned. This will allow the determination of sex differences in $\mathrm{MIH} / \mathrm{MH}$ prevalence and their potential interaction with other risk factors.

\section{CONCLUSION}

In conclusion, the prevalence rates of $\mathrm{MIH}$ and $\mathrm{MH}$ were determined to be $20.96 \%$ and $26.58 \%$, respectively, in 7- to 9year-old females in Makkah, Saudi Arabia. Both hypomineralization types were mainly mild in severity. There was no statistically significant association between any of the investigated etiological factors and MIH/MH. Nevertheless, additional studies are required to further elucidate the association of prenatal, perinatal and postnatal effects on hypomineralization. 


\section{ETHICS APPROVAL AND CONSENT TO PARTI- CIPATE}

The study received ethical approval from the Institutional Review Board (IRB) at the College of Dentistry, Umm AlQura University, Saudi Arabia (IRB No. 87-18).

\section{HUMAN AND ANIMAL RIGHTS}

No animals were used in this research. All human research procedures followed were in accordance with the ethical standards of the committee responsible for human experimentation (institutional and national), and with the Helsinki Declaration of 1975, as revised in 2013.

\section{CONSENT FOR PUBLICATION}

Parents of 865 children provided written informed consent.

\section{AVAILABILITY OF DATA AND MATERIALS}

All data that support the results of this study are restricted by the IRB, College of Dentistry, Umm Al-Qura University in order to protect patient privacy. Data can be made available upon reasonable request from the IRB secretary (irb.uqudent@uqu.edu.sa) only for researchers who meet the criteria for access to confidential data.

\section{FUNDING}

None.

\section{CONFLICT OF INTEREST}

The authors declare no conflict of interest, financial or otherwise.

\section{ACKNOWLEDGEMENTS}

Declared none.

\section{REFERENCES}

[1] Fagrell T. Molar incisor hypomineralization. Morphological and chemical aspects, onset and possible etiological factors. Swed Dent J Suppl 2011;216(216): 5-, 11-83 [PMID: 22338967]

[2] Weerheijm KL, Jälevik B, Alaluusua S. Molar-incisor hypomineralisation. Caries Res 2001; 35(5): 390-1. [http://dx.doi.org/10.1159/000047479] [PMID: 11641576]

[3] Preusser SE, Ferring V, Wleklinski C, Wetzel WE. Prevalence and severity of molar incisor hypomineralization in a region of Germany -a brief communication. J Public Health Dent 2007; 67(3): 148-50. [http://dx.doi.org/10.1111/j.1752-7325.2007.00040.x] [PMID: 17899899]

[4] de Lima MdeD, Andrade MJ, Dantas-Neta NB, et al. Epidemiologic study of molar incisor hypomineralization in school children in northeastern Brazil. Pediatr Dent 2015; 37(7): 513-9.

[PMID: 26883608]

[5] Saber F, Waly N, Moheb D. Prevalence of molar incisor hypomineralisation in a group of Egyptian children using the short form: a cross-sectional study. Eur Arch Paediatr Dent 2018; 19(5): 337-45.

[http://dx.doi.org/10.1007/s40368-018-0364-6] [PMID: 30178292]

[6] Soviero V, Haubek D, Trindade C, Da Matta T, Poulsen S. Prevalence and distribution of demarcated opacities and their sequelae in permanent $1^{\text {st }}$ molars and incisors in 7 to 13 -year-old Brazilian children. Acta Odontol Scand 2009; 67(3): 170-5. [http://dx.doi.org/10.1080/00016350902758607] [PMID: 19253064]

[7] Fteita D, Ali A, Alaluusua S. Molar-incisor hypomineralization (MIH) in a group of school-aged children in Benghazi, Libya. Eur Arch
Paediatr Dent 2006; 7(2): 92-5.

[http://dx.doi.org/10.1007/BF03320821] [PMID: 17140534]

[8] Zawaideh FI, Al-Jundi SH, Al-Jaljoli MH. Molar incisor hypomineralisation: prevalence in Jordanian children and clinical characteristics. Eur Arch Paediatr Dent 2011; 12(1): 31-6.

[http://dx.doi.org/10.1007/BF03262776] [PMID: 21299943]

[9] Elzein R, Chouery E, Abdel-Sater F, Bacho R, Ayoub F. Molar incisor hypomineralisation in Lebanon: prevalence and clinical characteristics. Eur Arch Paediatr Dent 2019; 20(6): 1-8. [PMID: 31865536]

[10] Ghanim A, Morgan M, Mariño R, Bailey D, Manton D. Molar-incisor hypomineralisation: prevalence and defect characteristics in Iraqi children. Int J Paediatr Dent 2011; 21(6): 413-21.

[http://dx.doi.org/10.1111/j.1365-263X.2011.01143.x]

[PMID: 21689174]

[11] Ofi WA, Salih BA. Prevalence and severity of molar-incisor hypomineralisation with relation to its etiological factors among school children 7-9 years of Al-Najaf governorate. J Baghdad Coll of Dent 2015; 27(3): 169-73.

[http://dx.doi.org/10.12816/0015053]

[12] Ditto D, Ajoor T, Alkhashram M, Alsharbati W. Prevalence of molar incisor hypomineralization in seven-nine-year-old Bahraini children an epidemiological study. J Bahrain Med Soc 2018; 30(1): 45-51.

[http://dx.doi.org/10.26715/jbms.1_26032018a]

[13] Hussain G, Al-Halabi M, Kowash M, Hassan A. The prevalence and severity of molar incisor hypomineralization and molar hypomineralization in Dubai, UAE. J Dent Child (Chic) 2018; 85(3): 102-7.

[PMID: 30869585]

[14] Allazzam SM, Alaki SM, El Meligy OA. Molar incisor hypomineralization, prevalence, and etiology. Int J Dent 2014; 2014 234508.

[http://dx.doi.org/10.1155/2014/234508] [PMID: 24949012]

[15] Al-Hammad NS, Al-Dhubaiban M, Alhowaish L, Bello LL. Prevalence and clinical characteristics of molar-incisorhypomineralization in school children in Riyadh, Saudi Arabia. IJMSCI 2018; 5(3): 3570-6.

[http://dx.doi.org/10.18535/ijmsci/v5i3.01]

[16] Alaluusua S. Aetiology of molar-incisor hypomineralisation: a systematic review. Eur Arch Paediatr Dent 2010; 11(2): 53-8. [http://dx.doi.org/10.1007/BF03262713] [PMID: 20403298]

[17] El Meligy OA, Alaki SM, Allazzam SM. Molar incisor hypomineralization in children: a review of literature. J Oral Hyg Health 2014; 2(4): 139-44.

[http://dx.doi.org/10.4172/2332-0702.1000139]

[18] Whatling R, Fearne JM. Molar incisor hypomineralization: a study of aetiological factors in a group of UK children. Int J Paediatr Dent 2008; 18(3): 155-62.

[http://dx.doi.org/10.1111/j.1365-263X.2007.00901.x] [PMID: 18384347]

[19] Weerheijm KL, Duggal M, Mejàre I, et al. Judgement criteria for molar incisor hypomineralisation (MIH) in epidemiologic studies: a summary of the European meeting on MIH held in Athens, 2003. Eur J Paediatr Dent 2003; 4(3): 110-3. [PMID: 14529329]

[20] Petrou MA, Giraki M, Bissar AR, et al. Prevalence of Molar-IncisorHypomineralisation among school children in four German cities. Int $\mathrm{J}$ Paediatr Dent 2014; 24(6): 434-40.

[http://dx.doi.org/10.1111/ipd.12089] [PMID: 24372870]

[21] Mittal NP, Goyal A, Gauba K, Kapur A. Molar incisor hypomineralisation: prevalence and clinical presentation in school children of the northern region of India. Eur Arch Paediatr Dent 2014; 15(1): 11-8.

[http://dx.doi.org/10.1007/s40368-013-0045-4] [PMID: 23754204]

[22] Elfrink ME, Ghanim A, Manton DJ, Weerheijm KL. Standardised studies on molar incisor hypomineralisation (MIH) and hypomineralised second primary molars (HSPM): a need. Eur Arch Paediatr Dent 2015; 16(3): 247-55.

[http://dx.doi.org/10.1007/s40368-015-0179-7] [PMID: 25894247]

[23] Jasulaityte L, Weerheijm KL, Veerkamp JS. Prevalence of molarincisor-hypomineralisation among children participating in the Dutch National Epidemiological Survey (2003). Eur Arch Paediatr Dent 2008; 9(4): 218-23.

[http://dx.doi.org/10.1007/BF03262638] [PMID: 19054475]

[24] Jälevik B. Prevalence and diagnosis of molar-incisorhypomineralisation (MIH): a systematic review. Eur Arch Paediatr Dent 2010; 11(2): 59-64. 
[http://dx.doi.org/10.1007/BF03262714] [PMID: 20403299]

[25] Jasulaityte L, Veerkamp JS, Weerheijm KL. Molar incisor hypomineralization: review and prevalence data from the study of primary school children in Kaunas/Lithuania. Eur Arch Paediatr Dent 2007; 8(2): 87-94

[http://dx.doi.org/10.1007/BF03262575] [PMID: 17555690]

[26] Al Agili DE. A systematic review of population-based dental caries studies among children in Saudi Arabia. Saudi Dent J 2013; 25(1): 3-11.

[http://dx.doi.org/10.1016/j.sdentj.2012.10.002] [PMID: 23960549]

[27] AlDosari AM, Akpata ES, Khan N. Associations among dental caries experience, fluorosis, and fluoride exposure from drinking water sources in Saudi Arabia. J Public Health Dent 2010; 70(3): 220-6. [http://dx.doi.org/10.1111/j.1752-7325.2010.00169.x] [PMID 20459462]

[28] Tapias-Ledesma MA, Jiménez R, Lamas F, González A, Carrasco P, Gíl de Miguel A. Factors associated with first molar dental enamel defects: a multivariate epidemiological approach. J Dent Child (Chic) 2003; 70(3): 215-20. [PMID: 14998204]

[29] Parikh DR, Ganesh M, Bhaskar V. Prevalence and characteristics of Molar Incisor Hypomineralisation (MIH) in the child population residing in Gandhinagar, Gujarat, India. Eur Arch Paediatr Dent 2012; 13(1): 21-6

[http://dx.doi.org/10.1007/BF03262836] [PMID: 22293101]
[30] Lygidakis NA, Chaliasou A, Siounas G. Evaluation of composite restorations in hypomineralised permanent molars: a four year clinical study. Eur J Paediatr Dent 2003; 4(3): 143-8. [PMID: 14529336]

[31] Taylor GD. Molar incisor hypomineralisation. Evid Based Dent 2017; 18(1): 15-6.

[http://dx.doi.org/10.1038/sj.ebd.6401219] [PMID: 28338027]

[32] Beentjes VE, Weerheijm KL, Groen HJ. Factors involved in the aetiology of molar-incisor hypomineralisation (MIH). Eur J Paediatr Dent 2002; 3(1): 9-13.

[PMID: 12871011]

[33] Silva MJ, Scurrah KJ, Craig JM, Manton DJ, Kilpatrick N. Etiology of molar incisor hypomineralization - A systematic review. Community Dent Oral Epidemiol 2016; 44(4): 342-53.

[http://dx.doi.org/10.1111/cdoe.12229] [PMID: 27121068]

[34] Tourino LF, Corrêa-Faria P, Ferreira RC, Bendo CB, Zarzar PM, Vale MP. Association between molar incisor hypomineralization in schoolchildren and both prenatal and postnatal factors: a populationbased study. PLoS One 2016; 11(6): e0156332.

[http://dx.doi.org/10.1371/journal.pone.0156332] [PMID: 27280451]

[35] Crombie F, Manton D, Kilpatrick N. Aetiology of molar-incisor hypomineralization: a critical review. Int J Paediatr Dent 2009; 19(2): 73-83.

[http://dx.doi.org/10.1111/j.1365-263X.2008.00966.x]

[PMID: 19250392]

\section{(C) 2021 Sulaimani et al.}

This is an open access article distributed under the terms of the Creative Commons Attribution 4.0 International Public License (CC-BY 4.0), a copy of which is available at: https://creativecommons.org/licenses/by/4.0/legalcode. This license permits unrestricted use, distribution, and reproduction in any medium, provided the original author and source are credited. 